\title{
SUSCETIBILIDADE DO CUPUAÇUZEIRO E OUTRAS ESPÉCIES VEGETAIS A ISOLADOS DE Crinipellis perniciosa OBTIDOS DE QUATRO HOSPEDEIROS DIFERENTES NO SUL DA BAHIA*
}

\author{
JOSÉ RONALDO M. LOPES, ${ }^{1}$ EDNA DORA M. N. LUZ ${ }^{* * *} \&$ JOSÉ LUIZ BEZERRA ${ }^{2 * *}$ \\ ${ }^{1}$ Centro de Extensão, CEPLAC, Cx. Postal 07, CEP 45.600-000, Itabuna-BA, fax: (073) 214-3325, \\ e-mail: setec@ ceplac.gov.br; ${ }^{2}$ Centro de Pesquisas, CEPEC/CEPLAC, Cx.Postal 07, CEP 45.600-000, \\ Itabuna-BA, fax (073) 214-3204, e-mail: sefit@ cepec.gov.br
}

(Aceito para publicação em 22/05/2001)

Autor para correspondência: José Ronaldo Monteiro Lopes

LOPES, J.R.M., LUZ, E.D.M.N. \& BEZERRA, J.L. Suscetibilidade do cupuçuazeiro e outras espécies vegetais a isolados de Crinipellis perniciosa obtidos de quatro hospedeiros diferentes no sul da Bahia. Fitopatologia Brasileira 26:601-605. 2001.

\section{RESUMO}

Visando avaliar as interações de suscetibilidade do cupuaçu (Theobroma grandiflorum) e de outros hospedeiros ao fungo Crinipellis perniciosa, plântulas de cupuaçu , cacau (Theobroma cacao), cacau-do-peru (Theobroma bicolor) e jurubeba (Solanum paniculatum), com idade entre seis a oito semanas, foram inoculadas com basidiósporos provenientes de vassouras secas e/ou frutos infetados destes hospedeiros coletados no sul da Bahia. As inoculações foram feitas depositando-se uma gota de $20 \mu \mathrm{l}$ da suspensão de 5,0 x $10^{5}$ basidiósporos $/ \mathrm{ml}$ de $C$. perniciosa, obtidos de cada um dos hospedeiros, na gema apical e no hipocótilo (cupuaçu) de cada muda. Após a inoculação as plântulas permaneceram por $24 \mathrm{~h}$ em câmara climatizada, com temperatura em torno de $25{ }^{\circ} \mathrm{C}$ e aproximadamente $100 \%$ de umidade. Realizou-se a avaliação final dos sintomas 60 dias após a inoculação. $\mathrm{O}$ delineamento experimental utilizado foi o inteiramente casualizado, com 20 tratamentos e quatro repetições de dez plantas. O cacau e o cacau-do-peru foram suscetíveis ao inóculo obtido dos quatro hospedeiros. A jurubeba apresentou reações de suscetibilidade somente aos inóculos dela própria e de cacau. $\mathrm{O}$ cupuaçu apresentou sintomas quando inoculado com basidiósporos obtidos dele próprio, de cacau e de cacaudo-peru. O inóculo proveniente de cacau foi o mais infetivo a todos os hospedeiros.

Palavras-chave: cacau, cacau-do-peru, jurubeba, Theobroma grandiflorum.

\section{ABSTRACT \\ Susceptibility of cupuassu and other plant species to isolates of Crinipellis perniciosa obtained from four different hosts in the South of Bahia}

Cross inoculations were performed to evaluate the reaction of cupuassu (Theobroma grandiflorum), cacao (Theobroma cacao), patashte (Theobroma bicolor) and jurubeba (Solanum paniculatum) to the inocula of Crinipellis perniciosa obtained from each of these host species. Basidiospores were obtained from basidiomata produced on dried brooms and/or diseased dried pods from each host collected in the south of Bahia. Inoculations were made by depositing a $20 \mu \mathrm{l}$ droplet of a $5 \times 10^{5}$ suspension of basidiospores of $C$. perniciosa on the terminal bud and on the hypocotile region (cupuassu) of each seedling. After inoculation the plants were kept in a moist chamber for $24 \mathrm{~h}$ under a temperature of $25 \pm 1{ }^{\circ} \mathrm{C}$ and at approximately $100 \%$ humidity. Symptoms were evaluated until 60 days after inoculation. The experimental design was completely randomized with 20 treatments and four replications of ten plants each. Cacao and patashte were susceptible to inocula obtained from all species used. Jurubeba was susceptible only to inoculum produced in its own species or in cacao. Cupuassu seedlings presented symptoms when inoculated with basidiospores produced from cupuassu, patashte and cacao. Inoculum obtained from cacao brooms was the most infective to all hosts.

\section{INTRODUÇÃO}

\footnotetext{
*Dissertação de Mestrado do primeiro autor apresentada à Universidade Federal da Bahia. (1999). Trabalho financiado pelo convênio CEPLAC/EMBRAPA/ SEAGRI/EBDA/FUNDECAU.

** Bolsistas do CNPq
}

A vassoura-de-bruxa, causada pelo fungo Crinipellis perniciosa (Stahel) Singer, é a mais importante doença do cacaueiro (Theobroma cacao L.), na Bahia, na Amazônia brasileira e nos demais países onde ocorre (Luz et al., 1997) 
e do cupuaçuzeiro [Theobroma grandiflorum (Willd. ex Spreng.) Schum.] na Amazônia.

Apesar do cacaueiro e cupuaçuzeiro estarem convivendo em áreas vizinhas na Bahia, onde a severidade no cacaueiro foi forte desde 1989 quando a doença foi registrada na região, somente em 1997, oito anos após seu aparecimento, foi feito o primeiro relato de sua ocorrência em cupuaçuzeiro no sul da Bahia.

Entre os hospedeiros de C. perniciosa, na Bahia, estão algumas plantas das famílias Sterculiaceae, Solanaceae e Malpighiaceae (Bezerra et al., 1998). Segundo Luz et al. (1997), naquele estado, os hospedeiros relacionados são: na família Sterculiaceae: T. cacao L., T. cf. sylvestris Aubl. ex Mart e Herrania spp; na família Solanaceae: Solanum paniculatum L. (jurubeba), S. gilo Raddi. (jiló), S. stipulaceum Willd. ex Roem \& Schutt (caiçara), S. melongena L. (beringela) e Solanum sp., além de Capsicum annuum L. (pimentão), C. frutescens L. (pimenta malagueta) e Athenaea cf. pogogena (Moric.) Sendt; na família Malpighiaceae apenas um cipó silvestre denominado Stigmaphyllon sp.

Até o momento, dentre os hospedeiros de C. perniciosa somente há confirmação de patogenicidade ao cacaueiro, na Amazônia e na Bahia, de isolados provenientes de jurubeba (Silva et al., 1992; Luz et al., 1997), A. cf. pogogena, uma solanácea silvestre sem nome vulgar (Bastos et al.,1991), além de Heteropterys acutifolia A. Juss., em Minas Gerais (Resende et al., 2000). Na amazônia, Bastos \& Evans (1985) e Bastos \& Andebrhan (1986), inoculando variedades de cacaueiro suscetíveis a vassoura-de-bruxa com inóculo obtido de solanáceas silvestres (Solanum. rugosum Rich. ex Poir. e $S$. laseantherum Heurck \& Muell. Arg.) e de Bixa orellana L., não conseguiram induzir sintomas no cacaueiro. Não se encontraram outros relatos na literatura de tentativas de inoculação para comprovação da patogenicidade ao cacaueiro de inóculos obtidos de outros hospedeiros, além daqueles que comprovaram a patogenicidade de inóculos provenientes de jurubeba e Athenaea cf. pogogena ao cacaueiro (Bastos et al., 1991; Silva et al.,1992). Vieira (1942) e Gonçalves (1965) sugeriram que as infecções no cacaueiro na Amazônia, poderiam ter se originado de populações do fungo associados ao cupuaçuzeiro que seria então o hospedeiro primário de $C$. perniciosa. No entanto, segundo Fonseca et al., (1985), Bastos (1986), Stein (1996) e Stein et al. (1996) isolados de cupuaçuzeiro não infetam cacau e vice-versa. Visando esclarecer a patogenicidade de isolados de cacaueiro e cupuaçuzeiro, a estes hospedeiros planejou-se testes de inoculação cruzada, incluindo isolados de jurubeba patogênicos ao cacaueiro (Silva et al., 1992), e também isolados de cacau-do-peru (Theobroma bicolor Humb. \& Bonpl.), hospedeiro de $C$. perniciosa, então recém constatado na Bahia (Lopes et al.,1999). Assim, objetivou-se avaliar se existe suscetibilidade cruzada a isolados de $C$. perniciosa entre quatro espécies de plantas (cupuaçu, cacau-do-peru, cacau e jurubeba). O termo isolado é utilizado neste trabalho para designar tanto as culturas puras quanto os basidiósporos obtidos de cada hospedeiro.

\section{MATERIAL E MÉTODOS}

Os experimentos foram conduzidos na Seção de Fitopatologia do Centro de Pesquisas do Cacau (CEPEC/ CEPLAC ) em Ilhéus, Bahia.

Sementes de cacau da cultivar Catongo (suscetível à vassoura-de-bruxa), cacau-do-peru e cupuaçu foram prégerminadas por $76 \mathrm{~h}$ em serragem curtida, esterilizada e umedecida, e plantadas em sacos de polietileno, com dimensões de $15 \mathrm{~cm}$ x $28 \mathrm{~cm}$ x $0,08 \mathrm{~cm}$, contendo $2 \mathrm{~kg}$ de solo esterilizado com brometo de metila. Dada a dificuldade de obter plântulas a partir de sementes de jurubeba em número suficiente para inoculação fez-se o enraizamento de estacas. Obtiveram-se hastes de $20 \mathrm{~cm}$ de comprimento e $1,5 \mathrm{~cm}$ de diâmetro, retiradas do caule principal de plantas de jurubeba, no campo, cortadas em bisel na base, onde aplicou-se o indutor de enraizamento, ácido indol butílico (AIB), na concentração de 4.000 ppm, utilizando talco como veículo. As estacas foram plantadas em sacos de polietileno com as dimensões e condições de terriço citadas anteriormente.

As plantas dos quatro hospedeiros foram cultivadas em condições de casa de vegetação por seis a oito semanas, de acordo com o período de desenvolvimento de cada espécie.

As sementes de cupuaçu e de cacau-do-peru foram obtidas de frutos provenientes de matrizes sadias e vigorosas da região sul da Bahia. As de cacau Catongo, foram provenientes de áreas comerciais do CEPEC/CEPLAC, e as mudas de jurubeba foram produzidas a partir de material colhido no município de Jequié-BA. Vassouras secas e frutos infetados dos hospedeiros em estudo, coletados em diversos municípios da região, foram colocados em vassoureiro (câmara indutora de formação de basidiocarpos) e submetidos a regime alternado de $8 \mathrm{~h}$ de molhamento e $16 \mathrm{~h}$ de secamento (Rocha \& Wheeler, 1985), para estimular a frutificação de C. perniciosa. Para coleta de basidiocarpos e obtenção de basidiósporos utilizou-se a metodologia descrita por Frias \& Purdy (1995). A porcentagem de germinação dos basidiósporos foi verificada imediatamente após a obtenção da suspensão e na véspera da inoculação. Somente as suspensões que apresentaram germinação superior a $80 \%$ foram usadas como inóculo.

As plântulas de todos os hospedeiros testados e as estacas de jurubeba, no estágio do segundo lançamento foliar, foram inoculadas com suspensões de $5 \times 10^{5}$ basidiósporos/ $\mathrm{ml}$ após terem suas folhas reduzidas a $1 / 3$ do tamanho, para acelerar o crescimento apical. $\mathrm{O}$ inóculo constou de uma gota com volume de $20 \mu \mathrm{l}$ da suspensão de basidiósporos, depositada na gema apical das plântulas. Em um segundo experimento, plântulas de cupuaçu foram inoculadas na região do hipocótilo, utilizando-se a mesma metodologia.

Após a inoculação, as plântulas permaneceram por $24 \mathrm{~h}$ em câmara climatizada, com temperatura em torno de $25 \pm 1{ }^{\circ} \mathrm{C}$ e umidade relativa do ar de aproximadamente $100 \%$, mantida por nebulizador, sendo então transferidas para a casa de vegetação, sob as condições normais de ambiente.

O delineamento experimental utilizado foi inteiramente casualizado, com 20 tratamentos (quatro hospedeiros 
inoculados com quatro inóculos diferentes) e quatro testemunhas (plântulas não inoculadas) de cada hospedeiro. Foram feitas quatro repetições de 10 plântulas cada, perfazendo um total de 40 plântulas por tratamento.

Realizaram-se observações semanais visando detectar o aparecimento dos primeiros sintomas para determinar o período de incubação, porém a avaliação final dos sintomas foi feita 60 dias após a inoculação. Foram observados os seguintes sintomas: vassouras terminais, axilares e cotiledonares e inchamento do caule, do hipocótilo, de pecíolos e dos pulvinos. A reação dos hospedeiros a cada isolado, foi determinada de acordo com a porcentagem de plantas infetadas, visando avaliar o grau de suscetibilidade. A cultivar Catongo foi usada como padrão de suscetibilidade ( Luz et al., 1999).

\section{RESULTADOS E DISCUSSÃO}

Entre os hospedeiros avaliados, submetidos à inoculação da gema apical na fase de plântula, somente o cacau e o cacau-do-peru apresentaram reações de suscetibilidade aos inóculos de $C$. perniciosa obtidos dos quatro hospedeiros testados (Tabela 1). Os sintomas nos dois hospedeiros foram visíveis na terceira semana após a inoculação. A jurubeba apresentou reações de suscetibilidade aos isolados de $C$. perniciosa provenientes de jurubeba e cacau, e o cupuaçu aos isolados de cupuaçu, cacau e cacau -do-peru. Os sintomas de vassoura-de-bruxa manifestaram-se com maior severidade no cacau (Figura 1a), seguido do cacau-do-peru (Figura 1b). Nestas duas espécies, verificaram-se maiores porcentagens de plantas infetadas (Tabela 1) e sintomas mais severos (Tabela 2). Devido à natureza qualitativa dos dados obtidos tornouse dispensável a sua análise estatística .

As plântulas de cupuaçu inoculadas no hipocótilo apresentaram, como reações de suscetibilidade, vassouras cotiledonares e inchamento do hipocótilo e da base da raiz principal (Figura 1c). As mudas de jurubeba exibiram hipertrofia leve quando inoculadas com basidiósporos provenientes de jurubeba e de cacau (Figura1d).

Cortes histológicos efetuados em algumas plantas, principalmete de jurubeba e cupuaçu, que apresentavam hiperplasia e hipertrofia leves após a infecção por $C$. perniciosa, revelaram a presença de hifas do patógeno nos tecidos dos hospedeiros.

Adicionalmente, encontrou-se também em algumas fazendas da região e na casa de vegetação do CEPEC, mudas de cupuaçu, entre quatro a seis meses de idade, com sintomas de vassoura-de-bruxa provenientes de infecção natural, devido à proximidade de área comercial de cacau severamente atacada. Mudas de cupuaçuzeiro inoculadas na parte apical com inóculos de cupuaçu e de cacau, apresentaram sintomas similares. Estas observações confirmam a suscetibilidade cruzada do cupuaçuzeiro a isolados de C. perniciosa provenientes do cacaueiro.

Neste estudo não ficou comprovada entretanto, a patogenicidade de $C$. perniciosa obtido de jurubeba a plântulas de cupuaçuzeiro, pois estas não apresentaram sintomas quando inoculadas tanto na gema apical como no hipocótilo. O mesmo ocorreu em gemas apicais de jurubeba quando inoculadas com inóculo de cupuaçu ou de cacau-do-peru. Barreto \& Evans (1996), propuseram a hipótese de que um ou mais hospedeiros alternativos de Crinipellis poderiam ter sido responsáveis pelo ingresso do patógeno em cacaueiros no estado da Bahia.

Stein (1996) não observou sintomas em cacaueiro e cupuaçuzeiro quando efetuou inoculação cruzada na gema apical de plântulas com disco de ágar contendo basidiósporos de $C$. perniciosa provenientes de cacaueiro e cupuaçuzeiro. No entanto, neste trabalho, usando como inóculo gotas da suspensão de basidiósporos na concentração recomendada por Nunes et al. (1996), observou-se a manifestação de sintomas nos dois hospedeiros, com inóculo obtido de ambos, e no cupuaçuzeiro usando inoculação na gema apical e no hipocótilo. Verificou-se maior porcentagem de plantas com sintomas nas inoculações feitas no hipocótilo, onde os sintomas foram bem distintos, com o inchamento localizado abaixo dos nós cotiledonares e formação de vassouras cotiledonares (Figura 1c).

A dificuldade de infecção do $C$. perniciosa na gema

TABELA 1 - Porcentagem de plântulas de cacua (Theobromae cacao), cacau-do-peru (Theobroma bicolor), cupuaçu (Theobroma grandiflorum) e jurubeba (Solanum paniculatum) com sintomas e tipos predominantes de sintomas quando inoculadas com diversos inóculos de Crinipellis perniciosa

\begin{tabular}{|c|c|c|c|c|c|c|c|c|c|}
\hline \multirow{3}{*}{ Origem do Inóculo } & \multirow{3}{*}{ APS 1} & & & \multicolumn{4}{|c|}{ Hospedeiros } & \multirow{2}{*}{\multicolumn{2}{|c|}{ Jurubeba }} \\
\hline & & \multicolumn{2}{|c|}{ Cacau } & \multicolumn{2}{|c|}{ Cacau-do-peru } & \multicolumn{2}{|c|}{ Cupuaçu } & & \\
\hline & & $\% \mathrm{I}^{2}$ & $\mathbf{T S}^{3}$ & $\% \mathbf{I}$ & TS & $\% \mathrm{I}$ & TS & $\% \mathrm{I}$ & TS \\
\hline Cacau & $3-4$ & 100,0 & $\mathrm{HV}^{4}$ & 70,0 & $\mathrm{HV}$ & 44,6 & $\mathrm{H}^{6}$ & 13,3 & $\mathrm{H}$ \\
\hline Cacau-do-peru & $3-4$ & 92,5 & $\mathrm{HV}$ & 80,0 & $\mathrm{HV}$ & 33,3 & $\mathrm{H}$ & - & - \\
\hline Cupuaçu & $6-7$ & 95,0 & $\mathrm{HV}$ & 92,5 & HV & 53,3 & $\mathrm{H}$ & - & - \\
\hline Jurubeba & $7-8$ & 90,0 & HV & 52,5 & HV & -5 & - & 26,6 & $\mathrm{H}$ \\
\hline
\end{tabular}

${ }^{1}$ APS=Aparecimento dos primeiros sintomas (semanas após a inoculação)

${ }^{2} \mathrm{I}(\%)=\%$ de plantas com sintomas: calculada em função do número total de plântulas inoculadas.

${ }^{3} \mathrm{TS}=$ Tipos de sintomas

${ }^{4} \mathrm{HV}=$ Inchamento e vassouras

${ }^{5}(-)=$ Plantas sem sintomas

${ }^{6} \mathrm{H}=$ Inchamento 
J.R.M. Lopes et al.

TABELA 2 - Freqüência (\%) de sintomas de vassoura-de-bruxa obtidos em quatro hospedeiros nas inoculações com diversos inóculos de Crinipellis perniciosa

\begin{tabular}{|c|c|c|c|c|c|c|c|c|}
\hline \multirow{3}{*}{ Hospedeiro } & \multicolumn{8}{|c|}{ Origem do inóculo } \\
\hline & \multicolumn{2}{|c|}{ Cacau-do-peru } & \multicolumn{2}{|c|}{ Cacau } & \multicolumn{2}{|c|}{ Cupuaçu } & \multicolumn{2}{|c|}{ Jurubeba } \\
\hline & $\% \mathrm{VV}^{1}$ & $\% \mathrm{HL}^{2}$ & $\% \mathrm{VV}$ & $\% \mathrm{HL}$ & $\% \mathrm{VV}$ & $\% \mathrm{HL}$ & $\% \mathrm{VV}$ & $\% \mathrm{HL}$ \\
\hline Cacau-do-peru & 84,3 & 15,7 & 96,4 & 3,6 & 97,3 & 2,7 & 95,2 & 4,8 \\
\hline Cacau & 94,7 & 5,3 & 100,0 & - & 100,0 & - & 100,0 & - \\
\hline Cupuaçu & 100,0 & - & 100,0 & - & 100,0 & - & - & - \\
\hline Jurubeba & -3 & - & - & 100,0 & - & - & - & 100,0 \\
\hline
\end{tabular}

${ }^{1} \mathrm{VV}(\%)=$ Porcentagem de plântulas com inchamento e proliferação com formação de vassouras

${ }^{2} \mathrm{HL}(\%)=$ Porcentagem de plântulas com inchamento.

${ }^{3}$ (-) Não houve expressão de sintomas

apical de mudas de cupuaçuzeiro pode ser devida à grande quantidade de pelos foliares (pó ferrugíneo) existentes. Verificou-se também demora na manifestação dos sintomas em comparação aos outros hospedeiros, o que pode ser devido ao desenvolvimento mais lento das mudas de cupuaçu. Vale destacar que as plântulas inoculadas no hipocótilo somente começaram a apresentar sintomas seis a sete semanas após a inoculação, com um período de incubação maior que nos outros hospedeiros (Tabela 1). Essas dificuldades podem ter impedido Fonseca et al. (1985), Bastos (1986), Stein (1996) e Stein et al. (1996), de obter infecções em cupuaçu levandoos a afirmar que isolados de cacaueiro não infetariam o cupuaçuzeiro e vice-versa. Os resultados deste trabalho, no entanto, contrariam essa afirmativa (Tabelas 1 e 2) suportando as afirmativas de Vieira (1942) e Gonçalves (1965).

Confirmou-se a patogenicidade dos isolados de $C$. perniciosa de jurubeba à cacau, conforme obtido por Silva et al. (1992). As provas de patogenicidade realizadas indicaram a provável resistência da jurubeba aos isolados de $C$. perniciosa oriundos do cupuaçu e cacau-do-peru, visto que aquela espécie não apresentou os sintomas característicos de vassoura-de-bruxa. Não há relatos na literatura de infecções em jurubeba com outro inóculo que não o proveniente do cacaueiro (Silva et al., 1992). É interessante notar que o isolado originário de $S$. paniculatum também não foi patogênico ao cupuaçu. Os sintomas manifestados em mudas de jurubeba inoculadas com basidiósporos obtidos de cacau e da própria jurubeba, na fase de plântula, não foram tão característicos da doença, o entumescimento foi leve e a presença de $C$. perniciosa só foi realmente comprovada por meio de cortes histológicos. Pode ser que seja necessário um maior período de tempo ou uma concentração mais alta de inóculo, para manifestação mais nítida dos sintomas.

Registrou-se neste experimento também a alta patogenicidade de isolados obtidos do cacau-do-peru ao cacaueiro e vice-versa, sendo este um fato novo na literatura, visto que, até então, inoculações cruzadas com inóculo obtido dos dois hospedeiros não haviam sido realizadas. Quase todas as plantas de cacau e cacau-do-peru apresentaram formação de vassouras terminais no período de 21 dias após a inoculação, com inóculo obtido dos quatro hospedeiros. É portanto recomendável a erradicação da jurubeba e do cacau-do-peru,

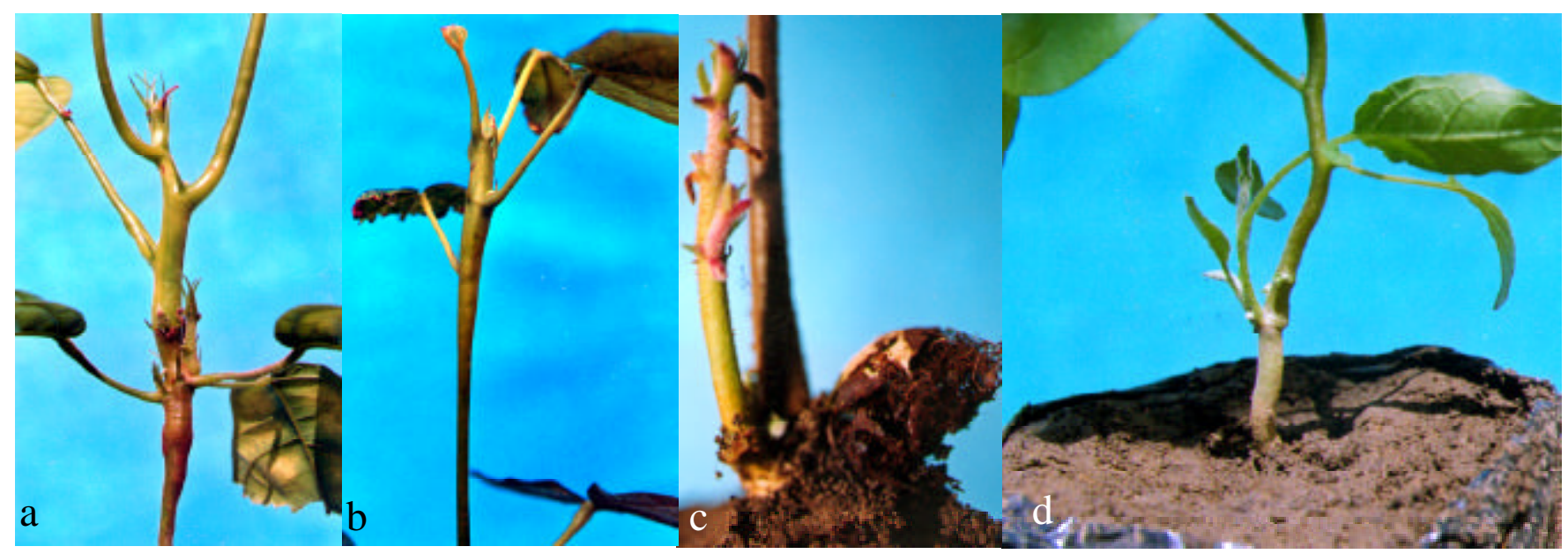

FIG. 1 - Sintomas de vassoura-de-bruxa em plântulas inoculadas com Crinipellis perniciosa provenientes de: a) plântulas de cacau (Theobromae cacao) com isolado de cupuaçu (Theobroma grandiflorum); b) de cacau-do-peru (Theobroma bicolor) com isolado de cacau; c) de cupuaçu com isolado de cacau-do-peru; e d) jurubeba (Solanum paniculatum) com isolado de cacau. 
nas proximidades de áreas comerciais de cupuaçu e cacau, por serem importantes fontes de inóculo de C. perniciosa.

\section{AGRADECIMENTOS}

À Comissão Executiva do Plano da Lavoura Cacaueira (CEPLAC), à Universidade Federal da Bahia - Escola de Agronomia, ao Convênio CEPLAC/EMBRAPA/SEAGRI/ EBDA/FUNDECAU, pelo financiamento do projeto e concessão da bolsa de incentivo à pesquisa ao primeiro autor, à Ana Rosa Cerqueira, Cenilda Rocha, Denise Argolo Ferreira, Márcia Paim e Virginia Damaceno, pela ajuda nos trabalhos de inoculação e avaliação dos sintomas

\section{REFERÊNCIAS BIBLIOGRÁFICAS}

BARRETO, R.W. \& EVANS, H.C. Duas novas solanáceas selvagens hospedeiras de Crinipellis perniciosa em Minas Gerais:uma explicação alternativa para a chegada da vassoura-de-bruxa do cacau na Bahia? Fitopatologia Brasileira 21:348.1996 (Resumo).

BASTOS, C.N. Comparação morfológica e patológica de isolados de Crinipellis perniciosa (Stahel) Singer. In: Informe técnico. Belém, PA. 1986. pp 45-49.

BASTOS, C.N. \& ANDEBRHAN, T. Urucu (Bixa orellana):nova espécie hospedeira da vassoura-de-bruxa (Crinipellis perniciosa) do cacaueiro. Fitopatologia Brasileira 11:963-965. 1986.

BASTOS, C.N. \& EVANS, H.C. A new pathotype of Crinipellis perniciosa. (Witches'broom) on solanaceous hosts. Plant Pathology 3:306-312. 1985.

BASTOS, C.N., SILVA, S.D.V.M. \& ALMEIDA, O.C. Ocorrência de vassoura-de-bruxa em solanácea silvestre na região cacaueira da Bahia. Agrotrópica 3:109-110. 1991.

BEZERRA, J.L., ALMEIDA, O.C. de \& SILVA, S.D.V.M. Hospedeiros colaterais de C. perniciosa na Bahia, Brasil. Fitopatologia Brasileira 23:228.1998 (Resumo).

FONSECA, S.E.A., ALMEIDA, L.C. de \& ANDEBRHAN, T. Patogenicidade de isolados e avaliação de resistência de clones de cacau a Crinipellis perniciosa. In: Anais, $9^{\text {a. }}$, International Cocoa Research Conference, 1984, Lomé, Lagos:Cocoa producers'Alliance, 1985. pp. 233-236.

FRIAS, G.A. \& PURDY, L.H. An inoculation method for resistance on cacao to Crinipellis perniciosa. Plant Disease 79:787-791. 1995.

GONÇALVES, J.R.C. Theobroma grandiflorum (Spreng.) Schum. as source of inoculum of witches' broom disease of Theobroma cacao L. Tropical Agriculture 42:261263. 1965.

LOPES, J.R.M., BEZERRA, J.L. \& LUZ, E.D.M.N. Theobroma bicolor hospedeiro de Crinipellis perniciosa na região sul da Bahia. Agrotrópica 11:97-100. 1999.
LUZ, E.D.M.N., BEZERRA, J.L., RESENDE, M.L.V. de \& OLIVEIRA, M.L de. Doenças do cacaueiro. In:Vale, F.X.R. \& Zambolim, L. (Eds.) Controle de doenças de plantas. Viçosa:UFV/Imprensa Universitária, 1997. pp. 611-616.

LUZ, E.D.M.N., SILVA, E.D.V.M., ALBUQUERQUE, P.S.B., GRAMACHO, K.P., BRUGNEROTTO, M.I.B., PAIM, M.C.A., PINTO, L.R.M., LOPES, U.V. \& PIRES, J.L. Evaluation of cacao progenies in Bahia for resistance to Crinipellis perniciosa. In: XII Conferência Internacional de Pesquisas do Cacau, 1999. Salvador. Proceedings of the XII International Cocoa Research Conference. Lagos-Nigéria:Cocoa Producers' Alliance, 1999. pp. 219-226.

NUNES, A.M.L., ALBUQUERQUE, F.C. de, OLIVEIRA, R.P. de, SÁ, T.D. de A., NUNES, M.A.L. \& SHIMIZU, O. Epidemiologia da vassoura-de-bruxa do cupuaçuzeiro. In: EMBRAPA. Centro de Pesquisa Agroflorestal da Amazônia Oriental. Geração de tecnologia agroindustrial para o desenvolvimento do trópico úmido. Belém: EMBRAPA-CPATU/JICA, Documentos, 85, 1996. pp. 83-105.

RESENDE, M.L.V., NOJOSA, G.B.A., SILVA, L.H.C.P., NIELLA, G.R., CARVALHO, G.A., SANTIAGO, D.V.R. \& BEZERRA, J.L. Crinipellis perniciosa proveniente de um novo hospedeiro, Heteropterys acutifolia é patogênico ao cacaueiro. Fitopatologia Brasileira 10:88. 2000.

ROCHA, H.M. \& WHEELER, B.E.J. Factor influencing production of basidiocarps and the deposition and germination of basidiospores of Crinipellis perniciosa, the causal fungus of witches'broom on cocoa (Theobroma cacao). Plant Pathology 34:319-328. 1985.

SILVA, S.D.V.M, GRAMACHO, K.P. \& ALMEIDA, O.C. de. Solanum paniculatum hospedeiro de Crinipellis perniciosa na região Sul da Bahia. Agrotrópica 4:1720. 1992.

STEIN, R.L.B. Vassoura-de-bruxa do cupuaçuzeiro ações de pesquisa e resultados. In: Anais, 1, Workshop sobre as Culturas de Cpuaçu e Pupunha na Amazônia, 1, 1996, Manaus. AM, EMBRAPA/CPAA, Documentos, 6, 1996. pp.149-157.

STEIN, R.L.B., ALBUQUERQUE, F.C. de, ROCHA NETO, O.G. da, CONCEIÇÃO, H.E.O da, MULLER, N.M., NUNUES, A.M.L., BASTOS, C.N., ENDO, T. \& ITO, S. Biologia e fisiologia de Crinipellis perniciosa do cupuaçuzeiro, em relação à fisiopatologia. In: EMBRAPA. Centro de Pesquisa Agroflorestal da Amazônia Oriental. Geração de tecnologia agroindustrial para o desenvolvimento do trópico úmido. Belém: EMBRAPA- CPATU/JICA, Documentos, 85, 1996. pp. 59-82.

VIEIRA, J.T. "Lagartão" ou "vassoura-de-bruxa”. Boletim da Sociedade Brasileira de Agronomia 5:393-400. 1942. 